\title{
Dipole induced transparency in waveguide coupled photonic crystal cavities
}

\author{
Andrei Faraon $^{1}$, Ilya Fushman ${ }^{1}$, Dirk Englund ${ }^{1}$, Nick Stoltz ${ }^{2}$, Pierre \\ Petroff $^{2}$, Jelena Vučković ${ }^{1}$ \\ ${ }^{1}$ E. L. Ginzton Laboratory, Stanford University, Stanford, CA, 94305 \\ ${ }^{2}$ Department of Electrical and Computer Engineering, University of California, Santa \\ Barbara, CA, 93106
}

\begin{abstract}
We demonstrate dipole induced transparency in an integrated photonic crystal device. We show that a single weakly coupled quantum dot can control the transmission of photons through a photonic crystal cavity that is coupled to waveguides on the chip. Control over the quantum dot and cavity resonance via local temperature tuning, as well as efficient out-coupling with an integrated grating structure is demonstrated.

(C) 2008 Optical Society of America

OCIS codes: (250.5300) Photonic integrated circuits; (270.1670) Coherent optical effects; (270.5580) Quantum electrodynamics; (270.5585) Quantum information and processing; (230.5298) Photonic crystals; (230.5750) Resonators;
\end{abstract}

\section{References and links}

1. D. Englund, A. Faraon, B. Zhang, Y. Yamamoto, and J. Vučković. "Generation and Transfer of Single Photons on a Photonic Crystal Chip," Opt. Express 15, (2007).

2. S. Noda, M. Fujita, and T. Asano. "Spontaneous-emission control by photonic crystals and nanocavities," Nat. Photonics 1, 449-458 (2007).

3. D. Englund, A. Faraon, I. Fushman, N. Stoltz, P. Petroff, and J. Vuckovic. "Controlling cavity reflectivity with a single quantum dot," Nature 450, 857-861 (2007).

4. A. Faraon, I. Fushman, D. Englund, N. Stoltz, P. Petroff, and J. Vuckovic, "Coherent generation of nonclassical light on a chip via photon-induced tunneling and blockade," arXiv:0804.2740v1 [quant-ph], (2008).

5. I. Fushman, D. Englund, A. Faraon, N. Stoltz, P. Petroff, and J. Vuckovic, "Controlled Phase Shifts with a Single Quantum Dot," Science 320, 769 - 772 (2008).

6. M. A. Nielsen and I. L. Chuang. Quantum Computation and Quantum Information, (Cambridge Univ. Press, Cambridge, 2000).

7. E. Waks and J. Vučković, "Dipole induced transparency in drop-filter cavity-waveguide systems," Phys. Rev. Lett. 96, 153601 (2006).

8. A. Auffèves-Garnier, C. Simon, J-M. Gérard, and J-P Poizat, "Giant optical nonlinearity induced by a single two-level system interacting with a cavity in the Purcell regime," Phys. Rev. A 75, 053823 (2007).

9. K. M. Birnbaum, A. Boca, R. Miller, A. D. Boozer, T. E. Northup, and H. J. Kimble, "Photon blockade in an optical cavity with one trapped atom," Nature 436, 87-90 (2005).

10. A. Högele, S. Seidl, M. Kroner, K.Karrai, R. J. Warburton, B. D. Gerardot, and P. M. Petroff, "Voltage-Controlled Optics of a Quantum Dot,” Phys. Rev. Lett. 93, 217401 (2004).

11. D. Haft, C. Schulhauser, A. Q. Govorov, R. J. Warburton, K. Karrai, J. M. Garcia, W. Schoedfeld, and P. M. Petroff, "Magneto-optical properties of ring-shaped self-assembled InGaAs quantum dots," Physica E 13, $165-169$ (2002).

12. A. Faraon, D. Englund, I. Fushman, N. Stoltz, P. Petroff, and J. Vuckovic, "Local quantum dot tuning on photonic crystal chips,” Appl. Phys. Lett. 90, 213110 (2007).

13. Y. Akahane, T. Asano, B.-S. Song, and S. Noda, "High-Q photonic nanocavity in a two-dimensional photonic crystal," Nature 425, 944-947 (2003).

14. A. Faraon, E. Waks, D. Englund, I. Fushman, and J. Vuckovic, "Efficient photonic crystal cavity-waveguide couplers,” Appl. Phys. Lett. 90, 073102 (2007).

\#97215 - \$15.00 USD

(C) 2008 OSA
Received 9 Jun 2008; revised 17 Jul 2008; accepted 17 Jul 2008; published 29 Jul 2008

4 August 2008 / Vol. 16, No. 16 / OPTICS EXPRESS 12154 


\section{Introduction}

The photonic crystal (PC) platform enables robust integration of optical resonators and quantum dots (QDs) into optical networks for classical and quantum information processing [1, 2]. Essential progress has been recently made with the demonstration of coherent probing of single QDs coupled to photonic crystal cavities[3], which opens the possibility of creating new optical devices where the flow of light is controlled via its interaction with the QD-resonator system. Two recent experiments demonstrate the potential of a QD-cavity system to generate nonclassical states of light and to mediate photon-photon interactions [4, 5], which is crucial for numerous quantum information processing applications [6].
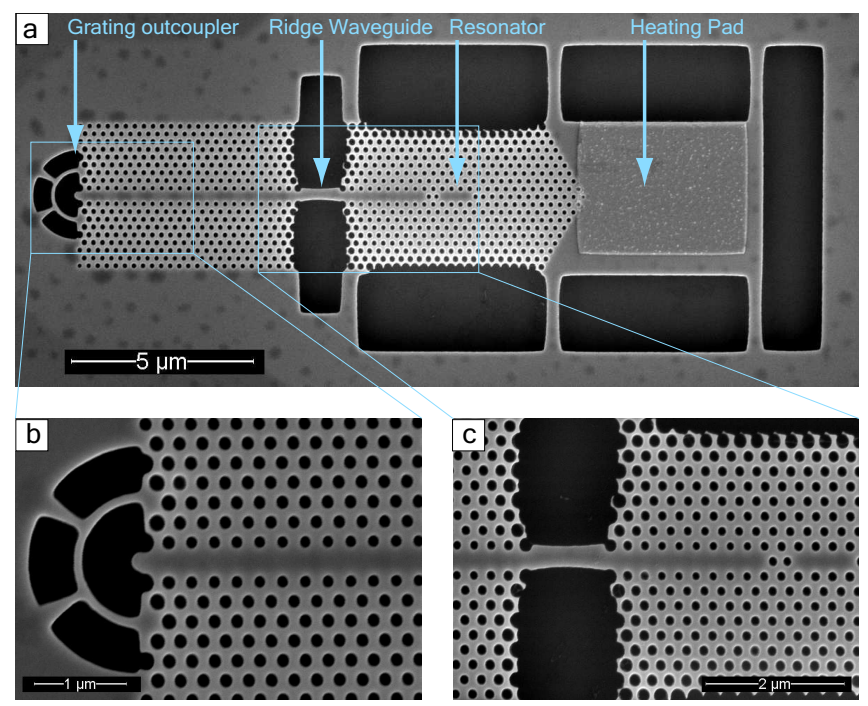

Fig. 1. (a) Photonic crystal device used to probe dipole induced transparency. The device consists of a PC cavity coupled to a PC waveguide terminated with a grating outcoupler. For local temperature control, the cavity is placed next to a metal pad that can be heated using an external laser beam. To increase the thermal insulation of the structure, the PC waveguide is interrupted and a narrow ridge waveguide link is inserted. (b) Magnified view of the grating outcoupler. (c) Magnified view of the ridge waveguide link

However, both of these experiments focused on QDs strongly coupled to cavities (i.e., the regime in which the QD-cavity field coupling strength $g$ is greater than $\kappa / 2$ where $\kappa$ is the cavity field decay rate). On the other hand, it has been theoretically predicted $[7,8]$ that a weakly coupled QD (with $g<\kappa / 2$ ) can also control the photon transmission through a resonator, as long as the system is in the strong Purcell regime $\left(g^{2} / \kappa \gamma>1\right.$, where $\gamma$ is the QD decay into modes other than the cavity mode). Such a regime is much easier to achieve in the solid state systems, as $\gamma<<g, \kappa$, as opposed to the atomic physics systems where $\kappa$ is on the same order as $\gamma$ [9]. This is especially important for the integrated structures, where cavities are coupled to waveguides, as such coupling degrades $\kappa$. We refer to such dipole assisted control of the photon transmission through a cavity as the dipole induced transparency [7, 8]; this effect can be classically explained as destructive interference at the output port of the PC cavity induced by the QD dipole. Thus, by controlling the state of the quantum dot one can change the cavity transmission function from transparent to opaque. The state of the quantum dot can be controlled either by shifting its resonance frequency via various effects such as the AC Stark shift [5], DC Stark shift [10], Zeeman shift [11], or by saturation [3, 5] using coherent laser beams 
or injected carriers.

\section{Device design and principle of operation}

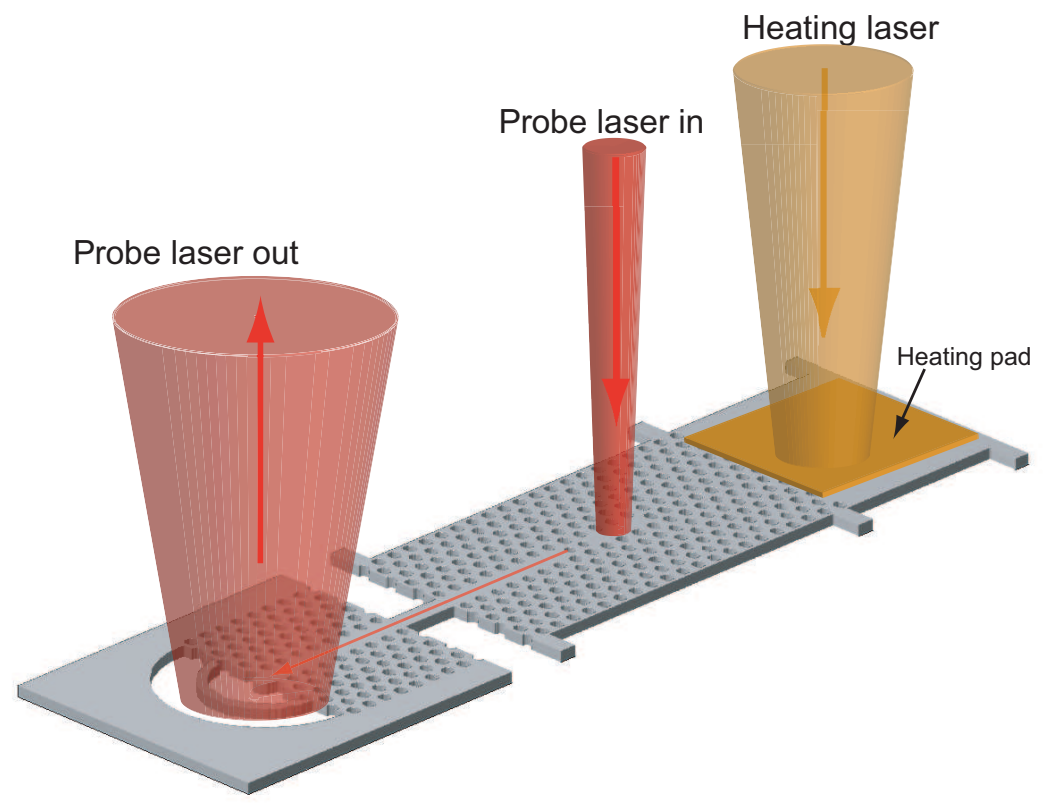

Fig. 2. Schematic showing the operation of the device. A heating laser is used to control the device temperature thus changing the resonance frequency of the cavity and the quantum dots coupled to it [12]. A probe laser is injected into the cavity from the top. The cavity field couples to the waveguide mode and then it is scattered from the grating outcoupler into the collection lens. A pinhole is used to collect only the output scattered by the grating. Using this device, the transmission function of the cavity can be analyzed for different frequencies of the resonator, quantum dot and probe laser.

In order to utilize the full potential of PCs and enable high fidelity, low off-chip loss operations, photonic information should be kept on the chip and outcoupled at the final information processing step. Such a scheme results in efficient cavity-waveguide coupling, enhanced signalto-noise ratios by separation of the input and output channels, and single mode operation, where a single cavity mode couples to a single waveguide mode. In this experiment we take the first step in this direction by designing a device that enables on-chip transmission measurements of PC cavities with coupled quantum dots.

To this end, we designed a photonic crystal device that integrates cavities and waveguides. For efficient light scattering out of the plane of the chip, the waveguide is terminated with a grating outcoupler. This allows us to measure the transmission of a probe beam injected into the cavity from the top, then coupled into the waveguide and outcoupled by the grating. The measurement principle is depicted in Fig. 2.

To achieve both large coupling efficiency into the waveguide and quality factors high enough 

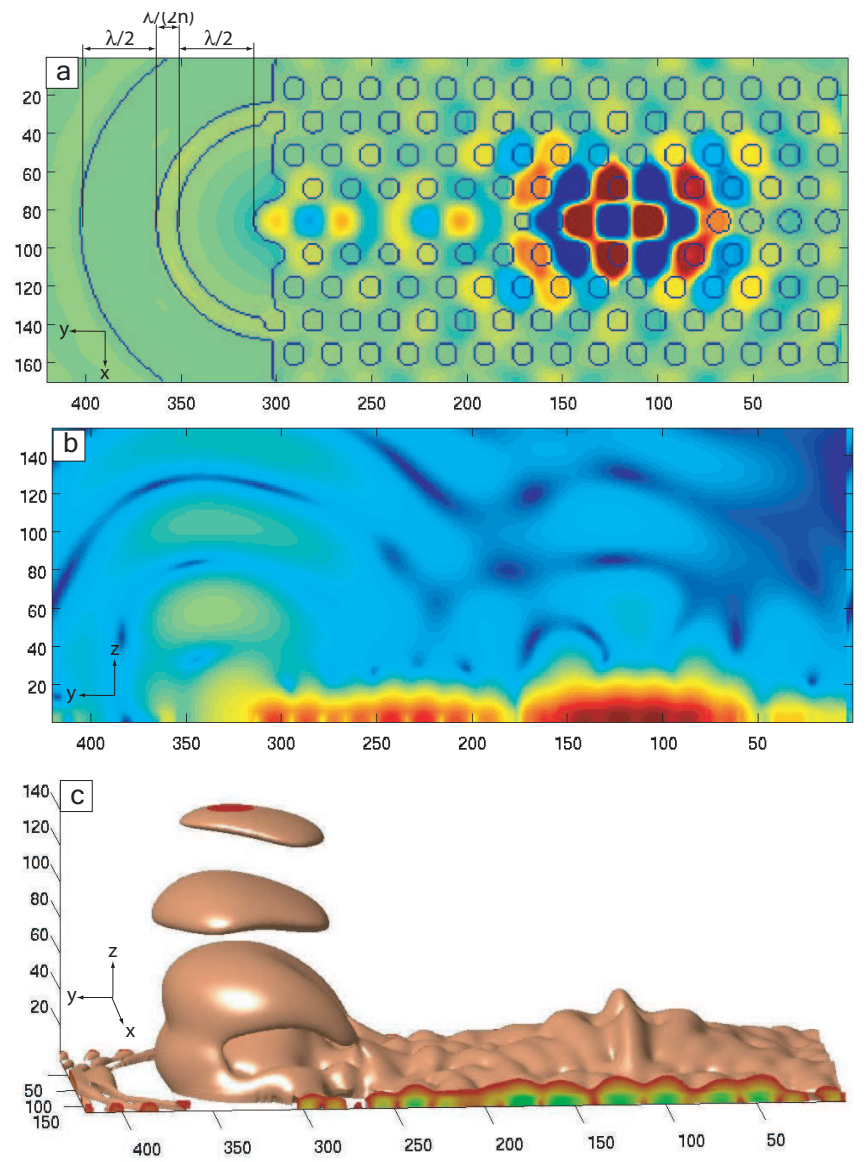

Fig. 3. Simulation of the L3 cavity field coupled into the waveguide whose output is vertically scattered by the grating outcoupler. (a) Magnetic field distribution in the plane of the photonic crystal, i.e., the x-y plane (the dominant, $B_{z}$ component is shown) (b) Energy density radiated from the structure, shown in the vertical cross-section through a plane passing through the middle of the waveguide and the cavity ( $\mathrm{x}-\mathrm{z}$ plane). Most of the vertically radiated energy is scattered from the grating outcoupler (c) Three dimensional view of one of the electromagnetic field density isosurfaces. This shows the profile of the evanescent cavity and waveguide field and indicates that most of energy radiated vertically comes from the grating outcoupler.

for strong Purcell enhancements, we choose a linear three-hole defect cavity (L3) [13] that is butt coupled to a PC waveguide with a two-hole separation between the cavity and the waveguide[14]. The device is further integrated with a metal heating pad that enables local temperature tuning of the QD and cavity resonance via external laser beams [12,3]. The suspended structure is connected to the rest of the substrate by only six narrow bridges to reduce the thermal conductivity [12]. One of the main thermally conductive elements is the photonic crystal waveguide. For increased thermal insulation we interrupted the photonic crystal waveguide and used a narrow ridge waveguide link.

To couple light from the photonic crystal waveguide out of the plane of the chip, we designed the grating outcoupler shown in Fig. 1(b). The grating outcoupler consists of a $\lambda /(2 n)$ pitch 
grating that causes destructive interference in the forward propagation direction and scatters most of the light into the collection lens. The grating is offset from the output port of the waveguide by $\lambda /(2 n)$ (Fig. 3(a)).

To verify the scattering properties of the grating, the device was simulated using the finite difference time domain method. Due to limited computing resources, a smaller scale structure than the one shown in Fig. 1 was simulated. The structure is shown in Fig. 3 (a) and consists of a L3 photonic crystal cavity butt coupled to a shorter PC waveguide that is directly terminated with the grating outcoupler. Figure 3(a) shows the distribution of the $z$ component of the magnetic field $\left(B_{z}\right)$, and shows how the cavity couples to the waveguide. In Figs. $3(\mathrm{~b}, \mathrm{c})$ we plot the energy density of the electromagnetic field, thus showing that the field scattered in the $z$ directions comes primarily from the grating. From the time evolution of the outcoupled field, we computed that $\sim 50 \%$ of the light outcoupled from the waveguide is scattered by the grating and collected into the microscope objective with 0.75 numerical aperture (NA) used in the experiment.

\section{Theoretical analysis}

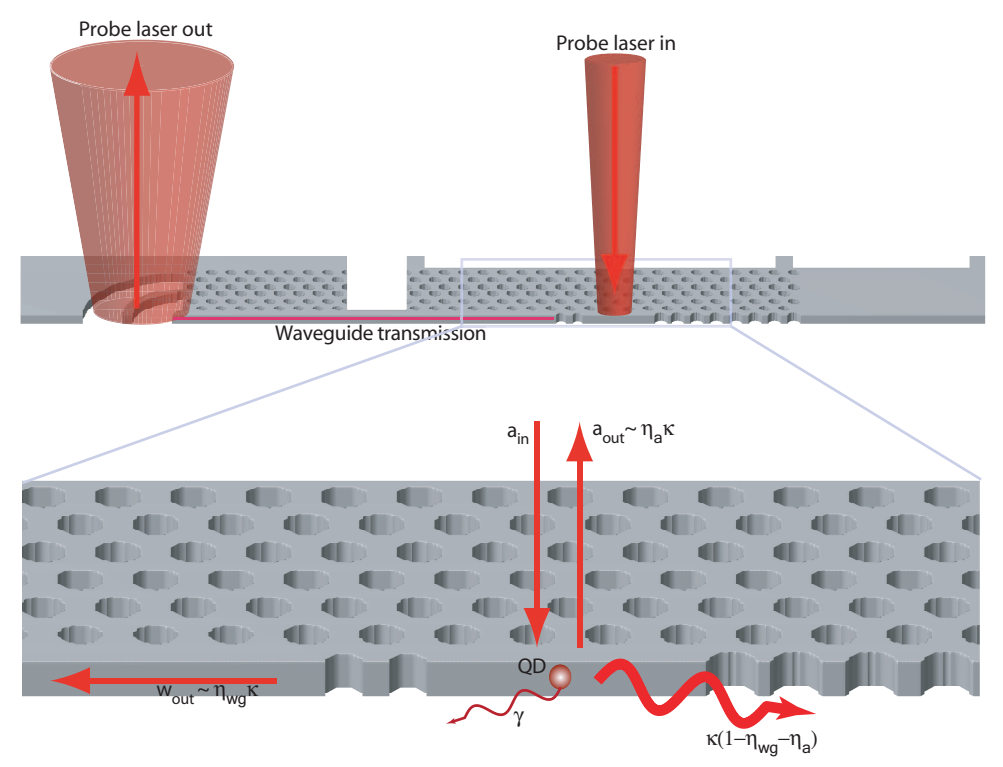

Fig. 4. Schematic of the cavity mode coupling into various photonic channels. The cavity couples with coupling constant $\eta_{a} \kappa$ to the forward and backward propagating modes $a_{i n}$ and $a_{\text {out }}$ of the probe beam. The other coupling channel of interest is the outward propagating waveguide mode $w_{\text {out }}$ with coupling rate to cavity equal to $\eta_{w g} \kappa$. The cavity loss into all other coupling channels is $\kappa\left(1-\eta_{w g}-\eta_{a}\right)$. Therefore, the total cavity field decay rate is $\kappa$. The uncoupled quantum dot decay rate is $\gamma$

Following a coupled mode theory formalism similar to the one described in Ref. [7], the transmission function of the system is described by the formula:

$$
T(\omega)=\left|\frac{w_{\text {out }}}{a_{\text {in }}}\right|^{2}=\left|\frac{2 \sqrt{\eta_{\text {wg }} \kappa} \sqrt{\eta_{a} \kappa}}{i\left(\omega_{c}-\omega\right)+\kappa+\frac{g^{2}}{i\left(\omega_{Q D}-\omega\right)+\gamma}}\right|^{2},
$$

\#97215 - \$15.00 USD

(C) 2008 OSA 
where $w_{\text {out }}$ is cavity emission outcoupled into the waveguide and $a_{\text {in }}$ is the mode of the lens used for coupling into the cavity. The coupling between the cavity and the quantum dot is $g$, the dipole decay rate without a cavity is $\gamma$, and $\omega_{c}, \omega_{Q D}, \omega$ are the resonance frequencies of the cavity, quantum dot and probe laser respectively. The total cavity loss $\kappa$ is divided into the loss into the waveguide $\eta_{w g} \kappa$, loss into the mode of the lens $\eta_{a} \kappa$, and loss into all the other channels $\kappa\left(1-\eta_{w g}-\eta_{a}\right)$ as shown schematically in Fig. 4.

To probe the dipole induced transparency we kept the probe laser constant and scanned the cavity and quantum dot resonance using local temperature tuning. During the local tuning process the cavity and quantum dot frequency shift linearly with the input power of the heating laser, with the quantum dot shifting approximately three times faster. The formula in Eq. 1 can be rewritten in terms of the power of the heating laser $(P)$ and the shifting rates of the cavity $\left(\alpha_{c}\right)$ and quantum $\operatorname{dot}\left(\alpha_{Q D}\right)$ with respect to the probe laser $\left(\alpha_{Q D} \sim 3 \alpha_{c}\right)$ :

$$
T(P)=\left|\frac{2 \sqrt{\eta_{w g} \kappa} \sqrt{\eta_{a} \kappa}}{i \alpha_{c} P+\kappa+\frac{g^{2}}{i \alpha_{Q D} P+\gamma}}\right|^{2},
$$

When no quantum dot is coupled to the cavity $(g=0)$ the transmission function $T(P)$ has a Lorentzian shape. The effect of a coupled quantum dot is a drop in the transmission function $[3,7]$. We stress that the transmission drop is not caused by absorption, but by the destructive interference at the output port caused by the interaction between the probe field, cavity field and the coherently driven quantum dot. While this effect is referred in literature as "dipole induced transparency" [7], in this particular configuration the dipole does not change the cavity transmission from opaque to transparent but the other way around (the quantum dot would induce transparency in a drop filter configuration [7]).

\section{Experimental implementation}

The device was fabricated on a quantum dot wafer grown by molecular beam epitaxy on a $\mathrm{Si}$ n-doped $\operatorname{GaAs}(100)$ substrate with a $0.1 \mu \mathrm{m}$ buffer layer, and a 10-period distributed Bragg reflector consisting of quarter-wave $\mathrm{AlAs} / \mathrm{GaAs}$ layers to improve collection efficiency into the lens. The distributed Bragg reflector is separated by a $918 \mathrm{~nm}$ sacrificial layer of $\mathrm{Al}_{0.8} \mathrm{Ga}_{0.2} \mathrm{As}$ from the $150-\mathrm{nm}$ GaAs membrane that contains a central layer of self-assembled InGaAs/GaAs quantum dots. The structure was fabricated using standard electron beam lithography, dry plasma etching and wet etching with hydrofluoric acid. To minimize the probability that more than one quantum dot couples to the same cavity mode, we used a sample with low quantum dot density ( $\sim 100$ quantum dots per $\left.\mu \mathrm{m}^{2}\right)$. The tuning pad was deposited as described in Ref.[12].

The sample was placed inside a continuous flow liquid helium cryostat and the measurements were performed using an experimental setup similar to that described in Ref. [3]. Using aboveband photoluminescence (PL) under weak excitation, we measured $Q=6140$ for the cavity quality factor, and a quantum dot weakly coupled to the cavity resonance was identified. The photoluminescence spectrum collected from the grating while locally tuning the temperature is shown in Figs. 5(a-d). Since the cavity-QD system is in the weak coupling regime, the spectrum does not show polariton anticrossing [12].

To characterize the grating outcoupler, a small aperture was used to collect photoluminescence either from the grating or the cavity. The collected spectra are plotted in Fig. 5(f). After background subtraction, the ratio between the grating collected PL and cavity collected PL was measured to be $P L_{\text {grat }} / P L_{c a v}=0.63$. Since for this cavity $Q=6140$, assuming a uncoupled quality factor of $Q_{c}=10000$ (similar to uncoupled cavities fabricated on the same chip) the $\mathrm{Q}$ corresponding to coupling into the waveguide can be deduced as $Q_{w g}=15900$ [14]. This implies that $\sim 39 \%$ of the cavity PL couples into the waveguide and $\sim 61 \%$ is radiated vertically

\#97215 - \$15.00 USD

(C) 2008 OSA
Received 9 Jun 2008; revised 17 Jul 2008; accepted 17 Jul 2008; published 29 Jul 2008 4 August 2008 / Vol. 16, No. 16 / OPTICS EXPRESS 12159 

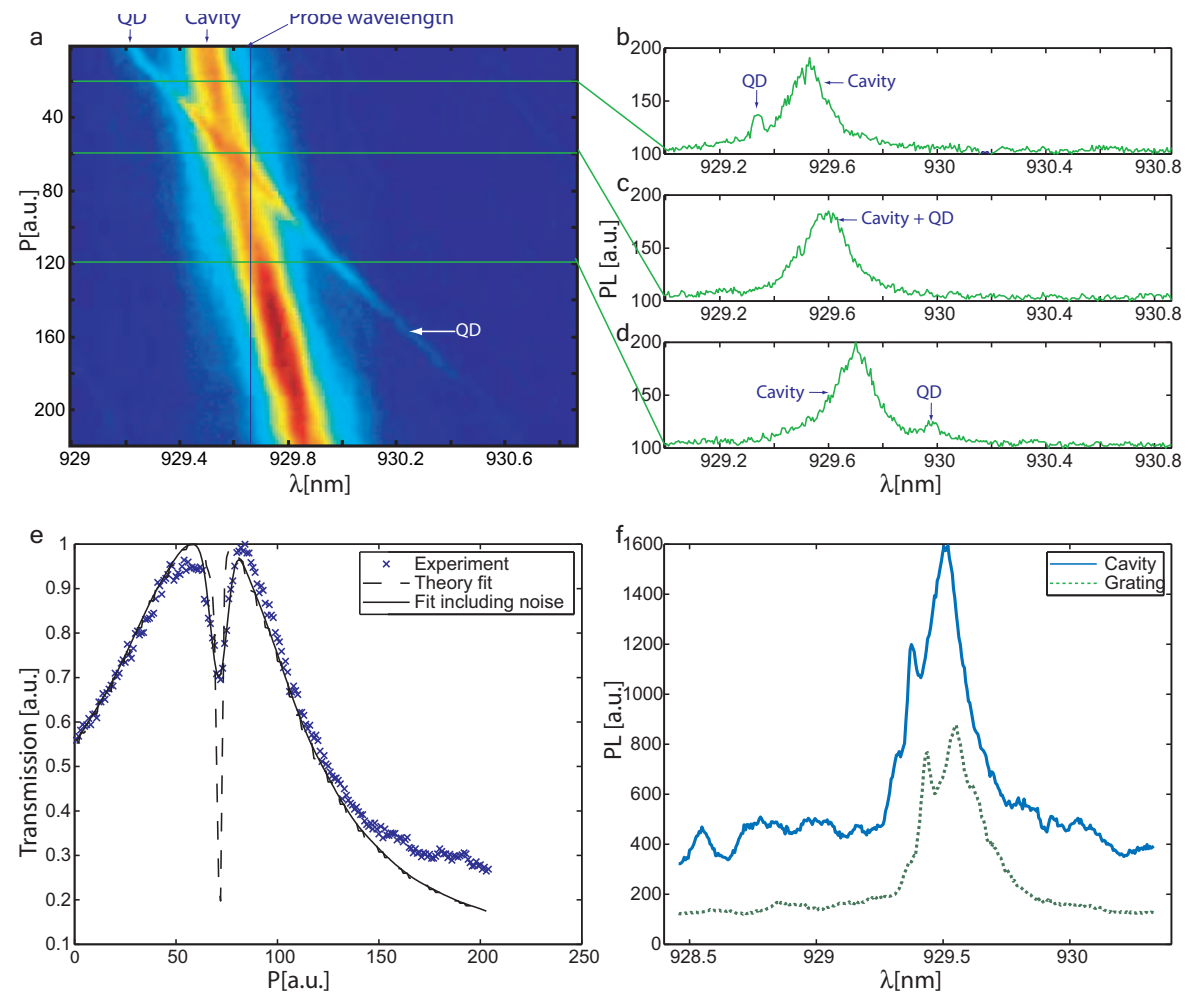

Fig. 5. (a) Two dimensional photoluminescence plot taken as the quantum dot is tuned into resonance with the cavity by changing the power of the heating laser (plotted on the vertical axis). (b-d) Photoluminescence plots at three different crossections marked by the horizontal lines in panel $a$. As expected for the weak coupling regime, the QD and the cavity lines cross. (e) Transmission measurement done by changing the power of the heating laser while the probe beam is kept fixed at the frequency marked by the vertical line in panel $b$. The plot shows the Lorentzian profile of the cavity resonance and the dipole induced transparency transmission dip induced by the quantum dot. The dashed line is the direct theoretical fit with Eq. 2. The solid line fit takes into account the fluctuations in the system. (f) Comparison between the photoluminescence spectra collected from the top of the cavity and the grating outcoupler. The two spectra were taken using a small aperture to collect only the photoluminescence from the area of interest. The ratio of the grating outcoupled cavity photoluminescence to cavity outcoupled photoluminescence is 0.64 .

(because of the reflecting DBR under the cavity), thus indicating a ratio of $P L_{\text {grat }} / P L_{\text {cav }}=0.64$ between the grating collected and cavity collected PL. This number is in excellent agreement with the one observed experimentally. However, we stress that despite the good matching the estimation is not completely accurate because the coupling losses at the PC/ridge waveguide interface and the coupling efficiencies into the numerical aperture of the lens were not taken into consideration. The losses in the system can only drive down the value for $P L_{g r a t} / P L_{c a v}$ observed experimentally, so the experimental result indicates that the grating radiates into the NA of the lens more efficiently than the cavity. This is in agreement with simulations which show that $\sim 50 \%$ of the light coupled into the waveguide is coupled into a lens with NA $=0.75$ while only $\sim 30 \%$ of the light radiated by the cavity is coupled into the same NA (these estimations 
take the back reflection of the DBR stack into consideration). The exact coupling efficiencies both form the grating and the cavity are currently under more careful characterization. Further design optimizations could lead to $P L_{\text {grat }}>>P L_{c a v}$, as desired.

The transmission measurements were performed using a focused diode laser (focal spot $\sim$ $1 \mu \mathrm{m}^{2}$ ) tuned to $929.65 \mathrm{~nm}$. The relative wavelength of the probe with respect to the cavity and quantum dot frequency is marked in Fig. 5(a). First, the coupling of the laser into the cavity was optimized using the cross-polarized reflectivity technique described in Ref.[3]. Once coupled, a small aperture was used to collect only the laser light transmitted from the cavity into the waveguide and then scattered by the grating. The experimental data for the device transmission is shown in Fig. 5(e). The data shows the Lorentzian transmission function of the cavity with a abrupt drop in transmission caused by dipole induced transparency with the weakly coupled quantum dot, as theoretically predicted [7]. To avoid quantum dot saturation, the experiment was performed at low probe power (tens of $\mathrm{nW}$ ) [3].
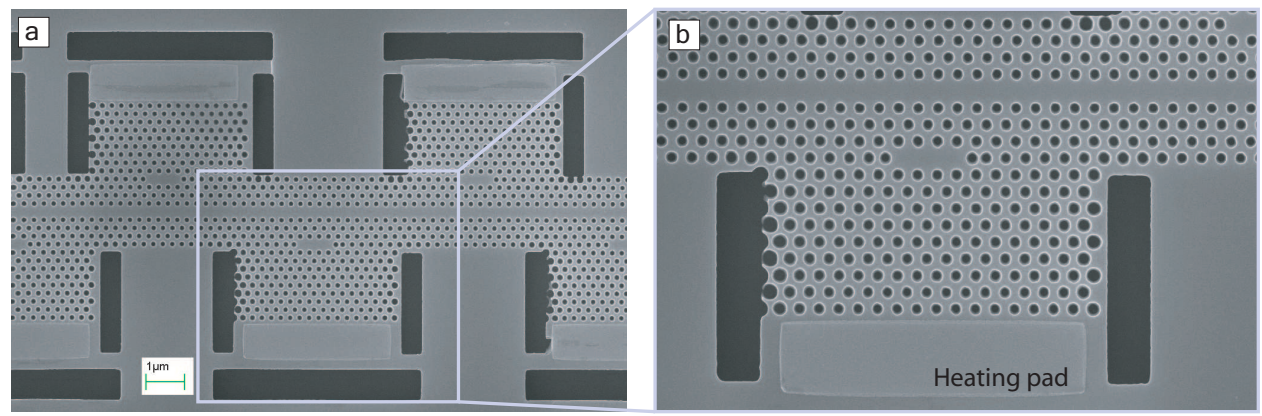

Fig. 6. (a) Prototype structure consisting the of photonic crystal resonators evanescently side coupled to a waveguide. Each resonator is next to a heating pad so the its temperature and thus its resonance frequency can be controlled independently. (b) Magnified view of the waveguide coupled resonator and its heating pad. The trenches surrounding the resonator provide local thermal insulation.

To compute the parameters of the system, the data was fit with Eq. 2 and the following values were obtained: $g / 2 \pi=9.4 \mathrm{GHz}, \kappa / 2 \pi=33 \mathrm{GHz}, \gamma / 2 \pi=0.3 \mathrm{GHz}$. The result of the theoretical fit using Eq.2 is shown in Fig. 5(e). As seen from the fit, our data is not fully described by Eq.2 because of various sources of noise in the system, mainly the temperature fluctuations due to small power fluctuations of the heating laser (which in turn induce wavelength fluctuations of the QD)[3]. When taking into account these fluctuations (as described in $[3,5]$ ), the solid line fit is obtained, in better agreement with the experimental data.

\section{Conclusion}

In conclusion, we observed dipole induced transparency in a photonic crystal device that integrates resonators, waveguides, outcouplers and local tuning elements. This shows that the coherent probing of coupled resonator-QD systems can be done not only in reflectivity but also in transmission measurements. In addition, the control of the light transmission through a resonator is done using a weakly coupled QD. The prototype device shown in this paper contains most of the building blocks of future quantum dot - photonic crystal networks for classical and quantum information processing. To increase the performance and functionality of future devices the coupling into the resonator should be done via another waveguide and more cavities and quantum dots should be interconnected. A prototype design of this kind is shown in Fig. 6. 


\section{Acknowledgments}

Financial support was provided by DARPA Young Faculty Award, ONR Young Investigator Award, the MURI Center for photonic quantum information systems (ARO/IARPA program No. DAAD19-03-1-0199) and NSF Grant No. CCF-0507295. Work was performed in part at the Stanford Nanofabrication Facility of NNIN supported by the National Science Foundation under Grant ECS-9731293.

\#97215 - \$15.00 USD

(C) 2008 OSA 